PROCEEDINGS OF THE

AMERICAN MATHEMATICAL SOCIETY

Volume 130, Number 1, Pages 111-120

S 0002-9939(01)06172-X

Article electronically published on June 8, 2001

\title{
SOME CHARACTERIZATIONS OF THE AUTOMORPHISMS OF $B(H)$ AND $C(X)$
}

\author{
LAJOS MOLNÁR
}

(Communicated by David R. Larson)

\begin{abstract}
We present some nonlinear characterizations of the automorphisms of the operator algebra $B(H)$ and the function algebra $C(X)$ by means of their spectrum preserving properties.
\end{abstract}

\section{INTRODUCTION}

Surjective linear maps between Banach algebras which preserve the spectrum are extensively studied in connection with a longstanding open problem sometimes called Kaplansky's problem on invertibility preserving linear maps. A weaker version of that problem reads as follows. Is it true that between semisimple Banach algebras every surjective linear map which preserves the spectrum is a Jordan homomorphism? For the algebra $B(X)$ of all bounded linear operators acting on a Banach space this was proved to be true by Jafarian and Sourour in [3]. As for commutative semisimple Banach algebras (for instance, the algebra $C(X)$ of all continuous complex functions on the compact Hausdorff space $X$ ) we once again have an affirmative answer to the question. Namely, in that case the result is a trivial consequence of the famous Gleason-Kahane-Żelazko theorem characterizing multiplicative linear functionals.

The aim of this paper is to investigate a similar problem omitting the condition of linearity. Clearly, nonlinear spectrum preserving transformations can be almost arbitrary. So, we have to impose a more restrictive condition. This will be the following: We assume that the spectrum of the product of the images of any two elements is equal to the spectrum of the product of those two elements. We shall see that on the studied algebras those transformations are "almost" automorphisms. Furthermore, we consider another preserving condition concerning the spectrum which will turn out to be in close relation to *-automorphisms. More precisely, the main results of the paper can be summarized as follows.

Received by the editors January 25, 2000 and, in revised form, May 31, 2000.

1991 Mathematics Subject Classification. Primary 47B49, 46J10.

Key words and phrases. Automorphism, operator algebra, function algebra, spectrum.

This research was supported by the Hungarian National Foundation for Scientific Research (OTKA), Grant No. T030082, T031995, and by the Ministry of Education, Hungary, Reg. No. FKFP 0349/2000. 
Main Theorem. Let $H$ be an infinite dimensional Hilbert space.

If $\phi: B(H) \rightarrow B(H)$ is a surjective function with the property that

$$
\sigma(\phi(A) \phi(B))=\sigma(A B) \quad(A, B \in B(H)),
$$

then $\phi$ is either an algebra automorphism or the negative of an algebra automorphism of $B(H)$.

If $\psi: B(H) \rightarrow B(H)$ is a surjective function with the property that

$$
\sigma\left(\psi(A)^{*} \psi(B)\right)=\sigma\left(A^{*} B\right) \quad(A, B \in B(H)),
$$

then $\psi$ is an algebra *-automorphism of $B(H)$ multiplied by a fixed unitary element.

If $H$ is finite dimensional, then in addition to the possibilities above we also get that $\phi$ can be an algebra antiautomorphism or the negative of an algebra antiautomorphism of $B(H)$ and $\psi$ can be an algebra ${ }^{*}$-antiautomorphism multiplied by a fixed unitary element.

If $X$ is a first countable compact Hausdorff space and $\phi: C(X) \rightarrow C(X)$ is a surjective function with the property that

$$
\sigma(\phi(f) \phi(g))=\sigma(f g) \quad(f, g \in C(X)),
$$

then $\phi$ is an algebra automorphism of $C(X)$ multiplied by a fixed continuous real function of modulus 1 .

If $\psi: C(X) \rightarrow C(X)$ is a surjective function with the property that

$$
\sigma(\overline{\psi(f)} \psi(g))=\sigma(\bar{f} g) \quad(f, g \in C(X)),
$$

then $\psi$ is an algebra (*-)automorphism of $C(X)$ multiplied by a fixed continuous complex function of modulus 1 .

The statement follows from the results of the paper which follow. We note that the referee kindly informed us about recent results on spectrum preserving maps which are not assumed to be linear; see [1] and also [2]. Furthermore, we remark that other nonlinear characterizations of the automorphisms of matrix algebras using preserving properties can be found in [9].

\section{Results}

We first fix the notation and definitions that we shall use throughout.

Every linear space is considered over the complex field. Let $X$ be a Banach space and denote by $B(X)$ the algebra of all bounded linear operators on $X$. The spectrum in any Banach algebra is denoted by $\sigma($.$) . In B(X)$, the spectrum has several important subsets. In what follows $\sigma_{p}(A)$ denotes the point spectrum of the operator $A \in B(X)$, that is,

$$
\sigma_{p}(A)=\{\lambda \in \mathbb{C}: A-\lambda I \text { is noninjective }\}
$$

and $\sigma_{s}(A)$ denotes the surjectivity spectrum of $A$, that is,

$$
\sigma_{s}(A)=\{\lambda \in \mathbb{C}: A-\lambda I \text { is nonsurjective }\} .
$$

If $x \in X$ and $f \in X^{*}$ ( $X^{*}$ is the dual space of $X$ ), then $x \otimes f$ stands for the operator of rank at most one defined by

$$
(x \otimes f)(y)=f(y) x \quad(y \in X) .
$$


Clearly, every finite rank operator $A \in B(X)$ is a finite linear combination of such operators. On the finite rank elements of $B(X)$ one can define the trace functional tr by

$$
\operatorname{tr} A=\sum_{n} f_{n}\left(x_{n}\right)
$$

where $A=\sum_{n} x_{n} \otimes f_{n}$. Then tr is a well-defined linear functional with the property that $\operatorname{tr}(T A)=\operatorname{tr}(A T)$ for every finite rank operator $A \in B(X)$ and for any $T \in$ $B(X)$. For a matrix $A \in M_{n}(\mathbb{C}), A^{t}$ denotes the transpose of $A$.

If $X$ is a compact Hausdorff space, then let $C(X)$ denote the algebra of all continuous complex valued functions on $X$. In this algebra the spectrum of an element equals its range. If $f \in C(X)$, then $\operatorname{supp} f$ stands for the support of $f$, that is, $\operatorname{supp} f=\overline{\{x \in X: f(x) \neq 0\}}$.

Turning to our results and their proofs, we remark that on operator algebras, besides linear maps preserving the spectrum one can also consider such transformations which preserve some parts of the spectrum. The following two results are of that type. In fact, they were motivated by [10, Theorem 3 and Theorem 4], respectively.

Theorem 1. Let $X$ be a Banach space and let $\phi: B(X) \rightarrow B(X)$ be a surjective function with the property that

$$
\sigma_{p}(\phi(A) \phi(B))=\sigma_{p}(A B) \quad(A, B \in B(X)) .
$$

If $X$ is infinite dimensional, then there is an invertible linear operator $T \in B(X)$ such that either

$$
\phi(A)=T A T^{-1} \quad(A \in B(X))
$$

or

$$
\phi(A)=-T A T^{-1} \quad(A \in B(X)) .
$$

If $\phi: M_{n}(\mathbb{C}) \rightarrow M_{n}(\mathbb{C})$ is a surjective function satisfying (1), then we have the following possibilities:

(a) there is an invertible matrix $T_{1} \in M_{n}(\mathbb{C})$ such that

$$
\phi(A)=T_{1} A T_{1}^{-1} \quad\left(A \in M_{n}(\mathbb{C})\right) ;
$$

(b) there is an invertible matrix $T_{2} \in M_{n}(\mathbb{C})$ such that

$$
\phi(A)=-T_{2} A T_{2}^{-1} \quad\left(A \in M_{n}(\mathbb{C})\right) ;
$$

(c) there is an invertible matrix $T_{3} \in M_{n}(\mathbb{C})$ such that

$$
\phi(A)=T_{3} A^{t} T_{3}^{-1} \quad\left(A \in M_{n}(\mathbb{C})\right)
$$

(d) there is an invertible matrix $T_{4} \in M_{n}(\mathbb{C})$ such that

$$
\phi(A)=-T_{4} A^{t} T_{4}^{-1} \quad\left(A \in M_{n}(\mathbb{C})\right) .
$$

Proof. We first show that $\phi$ is injective. Indeed, if $A, A^{\prime} \in B(X)$ are such that $\phi(A)=\phi\left(A^{\prime}\right)$, then from (11) we obtain that $\sigma_{p}(A B)=\sigma_{p}\left(A^{\prime} B\right)$ for every $B \in$ $B(X)$. This implies that

$$
\sigma_{p}(A x \otimes f)=\sigma_{p}\left(A^{\prime} x \otimes f\right) \quad\left(x \in X, f \in X^{*}\right) .
$$

It is an easy fact that if $\operatorname{dim} X \geq 2$, then

$$
\sigma_{p}(y \otimes g)=\{0, g(y)\} \quad\left(y \in X, g \in X^{*}\right) .
$$


Since in the one-dimensional case our statement is trivial, in what follows we assume that $\operatorname{dim} X \geq 2$. From (2) we infer that $f(A x)=f\left(A^{\prime} x\right)$ for every $x \in X, f \in X^{*}$. It follows that $A=A^{\prime}$ which proves the injectivity of $\phi$.

Observe that $\phi$ preserves the rank-one operators. In fact, this follows from the following characterization of rank-one elements of $B(X)$. The operator $A \in B(X)$ has rank one if and only if $A \neq 0,0 \in \sigma_{p}(T A)$ and $\# \sigma_{p}(T A) \leq 2$ for every $T \in B(X)$ (\# denotes cardinality). Observe that if $A \neq 0$, then $\phi(A) \neq 0$.

Our next step is to show that $\phi$ is linear. The easiest way to verify this is the use of the trace functional as follows. Since the trace of a rank-one operator $x \otimes f$ is $f(x)$, we obtain from (11) and (3) that

$$
\operatorname{tr} \phi(A) \phi(B)=\operatorname{tr} A B
$$

for every $A \in B(X)$ and rank-one operator $B \in B(X)$. If $A, A^{\prime} \in B(X)$ are arbitrary and $B \in B(X)$ is any rank-one operator, then we compute

$$
\begin{aligned}
& \operatorname{tr}\left(\left(\phi(A)+\phi\left(A^{\prime}\right)\right) \phi(B)\right)=\operatorname{tr} \phi(A) \phi(B)+\operatorname{tr} \phi\left(A^{\prime}\right) \phi(B) \\
& =\operatorname{tr} A B+\operatorname{tr} A^{\prime} B=\operatorname{tr}\left(A+A^{\prime}\right) B=\operatorname{tr} \phi\left(A+A^{\prime}\right) \phi(B) .
\end{aligned}
$$

By the arbitrariness of $B$ we obtain that $\phi$ is additive. One can check that $\phi$ is homogeneous in a similar way.

So, $\phi$ is a linear bijection of $B(X)$ preserving the rank-one operators. The form of such transformations is well-known. It follows from the argument in [4] leading to [4 Lemma 1.2] that we have two possibilities:

(i) there exist bijective linear operators $T: X \rightarrow X$ and $S: X^{*} \rightarrow X^{*}$ such that

$$
\phi(x \otimes f)=T x \otimes S f \quad\left(x \in X, f \in X^{*}\right) ;
$$

(ii) there exist bijective linear operators $T^{\prime}: X^{*} \rightarrow X$ and $S^{\prime}: X \rightarrow X^{*}$ such that

$$
\phi(x \otimes f)=T^{\prime} f \otimes S^{\prime} x \quad\left(x \in X, f \in X^{*}\right) .
$$

Suppose first that we have (i). According to (4) we obtain

$$
(S f)(T y) \cdot(S g)(T x)=f(y) g(x) \quad\left(x, y \in X, f, g \in X^{*}\right) .
$$

Consequently, there is a scalar $\lambda$ such that

$$
(S g)(T x)=\lambda g(x) \quad\left(x \in X, g \in X^{*}\right) .
$$

By the closed graph theorem we readily obtain that the bijective linear operators $T, S$ are bounded and hence we infer that $T^{*} S=\lambda I \in B\left(X^{*}\right)$. Thus, $S=\lambda T^{*-1}=$ $\lambda T^{-1^{*}}$ and this implies that $\phi(A)=\lambda T A T^{-1}$ for every finite rank operator $A \in$ $B(X)$. Using the property (1) of $\phi$ we have $\lambda^{2}=1$, that is, either $\lambda=1$ or $\lambda=-1$. Suppose that $\lambda=1$. Let $A \in B(X)$ be arbitrary. Pick any rank-one operator $\phi(B) \in B(X)$. From (4) it follows that

$$
\operatorname{tr} \phi(A) \phi(B)=\operatorname{tr} A B=\operatorname{tr}\left(T A T^{-1} T B T^{-1}\right)=\operatorname{tr}\left(T A T^{-1} \phi(B)\right) .
$$

By the arbitrariness of $\phi(B)$ we obtain that $\phi(A)=T A T^{-1}$ for every $A \in B(X)$.

Assume now that we have (ii). Similar to case (i) one can prove that $T^{\prime}: X^{*} \rightarrow X$ is a bounded invertible linear operator and

$$
\phi(x \otimes f)=\lambda T^{\prime}(f \otimes \tau(x)) T^{\prime-1} \quad\left(x \in X, f \in X^{*}\right)
$$

where $\tau$ denotes the natural embedding of $X$ into $X^{* *}$. Since $(x \otimes f)^{*}=f \otimes \tau(x)$, we obtain that in this case $\phi$ is of the form $\phi(A)=\lambda T^{\prime} A^{*} T^{\prime-1}$ for every finite rank 
operator $A \in B(X)$. Just as above, we infer that $\lambda= \pm 1$ and then obtain the form of $\phi$ on the whole $B(X)$. To see that in the infinite dimensional case this second possibility (ii) cannot occur, we refer to [10, Theorem 3] stating that on an infinite dimensional Banach space $X$ every point spectrum preserving surjective linear map is an automorphism (not an antiautomorphism). Since, as it can be seen, $\phi$ or $-\phi$ satisfies these conditions, that result applies.

To verify that the finite dimensional case is different, that is, (ii) can really occur, we remark that in that case the injectivity, surjectivity, bijectivity of an operator are all equivalent and that it is true for any elements $A, B$ of any Banach algebra that $\sigma(A B) \backslash\{0\}=\sigma(B A) \backslash\{0\}$. Consequently, for every $A, B \in M_{n}(\mathbb{C})$ we have $\sigma_{p}\left(A^{t} B^{t}\right)=\sigma_{p}\left((B A)^{t}\right)=\sigma_{p}(B A)=\sigma_{p}(A B)$. The proof is complete.

Considering the surjectivity spectrum we have a similar result which follows.

Theorem 2. Let $H$ be an infinite dimensional Hilbert space and let $\phi: B(H) \rightarrow$ $B(H)$ be a surjective function with the property that

$$
\sigma_{s}(\phi(A) \phi(B))=\sigma_{s}(A B) \quad(A, B \in B(H)) .
$$

Then there is an invertible linear operator $T \in B(H)$ such that either

$$
\phi(A)=T A T^{-1} \quad(A \in B(H))
$$

or

$$
\phi(A)=-T A T^{-1} \quad(A \in B(H)) .
$$

Proof. One can argue in a very similar way as in our first result. This can be done since, by the Fredholm alternative, for any finite rank operator (in fact, even for any compact operator) $A \in B(X)$ we have

$$
\sigma_{p}(A) \backslash\{0\}=\sigma(A) \backslash\{0\}=\sigma_{s}(A) \backslash\{0\} .
$$

To exclude the appearance of the second possibility (ii) in the proof of Theorem 1 choose a nonsurjective isometry $V$ on $H$. Let $A=V^{*}$ (the Banach space adjoint of $V$ ) and set $B=V$. Then we see that $A B$ is invertible while $A^{*} B^{*}$ is not surjective. So, $\sigma_{s}\left(A^{*} B^{*}\right) \neq \sigma_{s}(A B)$.

Using the same argument once again, we have the following result.

Theorem 3. Let $H$ be an infinite dimensional Hilbert space and let $\phi: B(H) \rightarrow$ $B(H)$ be a surjective function with the property that

$$
\sigma(\phi(A) \phi(B))=\sigma(A B) \quad(A, B \in B(H)) .
$$

Then there is an invertible linear operator $T \in B(H)$ such that either

$$
\phi(A)=T A T^{-1} \quad(A \in B(H))
$$

or

$$
\phi(A)=-T A T^{-1} \quad(A \in B(H)) .
$$

Now we turn to a similar characterization of *-automorphisms.

Theorem 4. Let $H$ be a Hilbert space and let $\phi: B(H) \rightarrow B(H)$ be a surjective function with the property that

$$
\sigma\left(\phi(A)^{*} \phi(B)\right)=\sigma\left(A^{*} B\right) \quad(A, B \in B(H)) .
$$


If $H$ is infinite dimensional, then there are unitary operators $U, V \in B(H)$ such that $\phi$ is of the form

$$
\phi(A)=U A V \quad(A \in B(H)) .
$$

If $\phi: M_{n}(\mathbb{C}) \rightarrow M_{n}(\mathbb{C})$ is a surjective funtion satisfying $(7)$, then there are unitaries $U, V \in M_{n}(\mathbb{C})$ such that $\phi$ is either of the form

$$
\phi(A)=U A V \quad\left(A \in M_{n}(\mathbb{C})\right)
$$

or of the form

$$
\phi(A)=U A^{t} V \quad\left(A \in M_{n}(\mathbb{C})\right) .
$$

Proof. The linearity of $\phi$ can be proved in a very similar way as above. Since the norm and the spectral radius of a selfadjoint operator in $B(H)$ are equal, it follows from $\sigma\left(\phi(A)^{*} \phi(A)\right)=\sigma\left(A^{*} A\right)$ that $\|\phi(A)\|^{2}=\|A\|^{2}(A \in B(H))$. Consequently, $\phi$ is a surjective linear isometry of $B(H)$. The form of such transformations is well-known. Namely, to every surjective linear isometry $\psi$ there exist unitaries $U, V \in B(H)$ such that $\psi$ is either of the form

$$
\psi(A)=U A V \quad(A \in B(H))
$$

or of the form

$$
\psi(A)=U A^{t} V \quad(A \in B(H)) .
$$

If $H$ is of infinite dimension, then the appearance of this second possibility can be excluded just as in the last part of the proof of Theorem 2,

We next treat our problems in the case of the function algebra $C(X)$ on a compact Hausdorff space $X$.

Theorem 5. Let $X$ be a first countable compact Hausdorff space. If $\phi: C(X) \rightarrow$ $C(X)$ is a surjective function with the property that

$$
\sigma(\phi(f) \phi(g))=\sigma(f g) \quad(f, g \in C(X)),
$$

then there exist a homeomorphism $\varphi: X \rightarrow X$ and a continuous function $\tau: X \rightarrow$ $\{-1,1\}$ such that

$$
\phi(f)(x)=\tau(x) f(\varphi(x)) \quad(x \in X, f \in C(X)) .
$$

Proof. We have $\sigma\left(\phi(1)^{2}\right)=\sigma(1)$. The spectrum of an element of $C(X)$ equals its range. Therefore, $\phi(1)^{2}=1$ and considering the transformation $f \mapsto \phi(1) \phi(f)$, we can and do assume that our function $\phi$ satisfies $\phi(1)=1$.

We obtain from (8) that $\sigma(\phi(f))=\sigma(f)$ for every $f \in C(X)$. So, $\phi$ preserves the range of functions. This implies that $\phi$ maps real functions to real functions and it sends nonnegative functions to nonnegative functions.

We prove that $\phi$ is injective. This will follow from the following characterization of the equality between functions. Let $f, g \in C(X)$. Then $f=g$ if and only if $\sigma(f h)=\sigma(g h)$ for every nonnegative function $h \in C(X)$. Indeed, suppose that $f\left(x_{0}\right) \neq g\left(x_{0}\right)$ for some $x_{0} \in X$. We can assume that $\left|f\left(x_{0}\right)\right| \leq\left|g\left(x_{0}\right)\right|$. Let $D$ be an open disk centered at $f\left(x_{0}\right)$ which does not contain $g\left(x_{0}\right)$ and let $U$ be a neighbourhood of $x_{0}$ such that $f(x) \in D$ for every $x \in U$. Let $h: X \rightarrow[0,1]$ be a continuous function such that $\operatorname{supp} h \subset U$ and $h\left(x_{0}\right)=1$. Such a function exists by Urysohn's lemma. Then we obtain $\sigma(f h) \subset[0,1] D$ but $g\left(x_{0}\right) h\left(x_{0}\right)=g\left(x_{0}\right) \notin$ $[0,1] D$. Therefore, $\sigma(f h) \neq \sigma(g h)$. So, we have the injectivity of $\phi$. Therefore, $\phi$ and $\phi^{-1}$ are bijective functions having the same properties concerning the spectrum. 
Our next claim is that $\phi$ preserves the usual ordering between real functions. This will follow from the following characterization of that ordering. If $f, g \in C(X)$ are real functions, then $f \leq g$ if and only if

(a) $h g \leq c \Longrightarrow h f \leq c$ for every $0 \leq h \in C(X)$ and $c \in \mathbb{R}$

and

(b) $h f \geq c \Longrightarrow h g \geq c$ for every $0 \leq h \in C(X)$ and $c \in \mathbb{R}$.

To see this, suppose that $f\left(x_{0}\right)>g\left(x_{0}\right)$ for some $x_{0} \in X$. Clearly, there exists a positive number $\epsilon$ such that either $f\left(x_{0}\right)$ does not belong to the $\epsilon$-neighbourhood of $\left[0, g\left(x_{0}\right)\right]$ or $g\left(x_{0}\right)$ does not belong to the $\epsilon$-neighbourhood of $\left[0, f\left(x_{0}\right)\right]$. Suppose that we have the first possibility. Choose a continuous function $h: X \rightarrow[0,1]$ for which $h\left(x_{0}\right)=1$, and the support of $h$ is a subset of a neighbourhood of $x_{0}$ in which $g$ takes its values in the $\epsilon$-neighbourhood of $g\left(x_{0}\right)$. Then we find that $\sigma(h g)$ is a subset of the $\epsilon$-neighbourhood of $\left[0, g\left(x_{0}\right)\right]$ but $\sigma(h f)$ is not a subset of that set. It is easy to see that there is a constant $c$ such that $h g \leq c$ but $h f \not \leq c$. So, the above characterization really holds and then we get that $f \leq g$ if and only if $\phi(f) \leq \phi(g)$.

Observe that by (8) we have $f g=0$ if and only if $\phi(f) \phi(g)=0$. Now, if $f, g \geq 0$ and $f g=0$, then we find that

$$
\phi(f+g)=\phi(\max \{f, g\})=\max \{\phi(f), \phi(g)\}=\phi(f)+\phi(g) .
$$

To any point $x \in X$ there exists a continuous function $f_{x}: X \rightarrow[0,1]$ such that $f_{x}(x)=1$ and $f_{x}(y)<1$ if $y \neq x$. In fact, by the first countability of $X$ there is a sequence $\left\{U_{n}\right\}$ of neighbourhoods of $x$ which forms a base of neighbourhoods of that point. For every $n \in \mathbb{N}$ there is a continuous function $f_{n}: X \rightarrow[0,1]$ whose support is in $U_{n}$ and $f_{n}(x)=1$. Now, set $f_{x}=\sum_{n} \frac{1}{2^{n}} f_{n}$. This function fulfills our requirements. Denote by $\mathcal{F}_{x}$ the set of all such function $f_{x}$. We assert that if $f_{x} \in \mathcal{F}_{x}$, then $\phi\left(f_{x}\right)$ belongs to $\mathcal{F}_{\varphi(x)}$ for some $\varphi(x) \in X$. In fact, since $\phi$ preserves the range of functions, it follows that $\phi\left(f_{x}\right)$ maps into $[0,1]$ and it takes the value 1. Suppose that $\phi\left(f_{x}\right)$ equals 1 at two different points, say $y, z$. It follows that there are functions $g^{\prime} \in \mathcal{F}_{y}$ and $h^{\prime} \in \mathcal{F}_{z}$ such that $g^{\prime} h^{\prime}=0$ and $g^{\prime}+h^{\prime} \leq \phi\left(f_{x}\right)$. Let $g=\phi^{-1}\left(g^{\prime}\right)$ and $h=\phi^{-1}\left(h^{\prime}\right)$. Then we have $g h=0$ and by the previous sections of the proof we infer that $g+h=\phi^{-1}\left(g^{\prime}+h^{\prime}\right) \leq f_{x}$. Since $g+h$ takes the value 1 at at least two points, the same must be true for $f_{x}$, which is a contradiction. This means that $\phi\left(f_{x}\right) \in \mathcal{F}_{\varphi(x)}$ for some $\varphi(x) \in X$. We next show that the point $\varphi(x)$ does not depend on the particular choice of $f_{x}$. Indeed, let $f_{x}, f_{x}^{\prime} \in \mathcal{F}_{x}$. Then $\max \left\{f_{x}, f_{x}^{\prime}\right\} \in \mathcal{F}_{x}$ and this implies that $\max \left\{\phi\left(f_{x}\right), \phi\left(f_{x}^{\prime}\right)\right\} \in \mathcal{F}_{y}$ for some $y \in X$. This proves that $\phi\left(f_{x}\right)$ and $\phi\left(f_{x}^{\prime}\right)$ take their maximum at the same point. So, we have a function $\varphi: X \rightarrow X$ such that $f_{x} \in \mathcal{F}_{x}$ implies $\phi\left(f_{x}\right) \in \mathcal{F}_{\varphi(x)}$. Since $\phi$ and $\phi^{-1}$ share the same properties, we obtain that $\varphi$ is a bijection.

We assert that $\phi$ is homogeneous. Let $f \in C(X)$ and $\lambda \in \mathbb{C}$. For any $0 \leq h \in$ $C(X)$ we have

$$
\sigma(\lambda \phi(f) \phi(h))=\lambda \sigma(\phi(f) \phi(h))=\lambda \sigma(f h)=\sigma((\lambda f) h)=\sigma(\phi(\lambda f) \phi(h))
$$

which implies that $\phi(\lambda f)=\lambda \phi(f)$.

Let $0 \leq f \in C(X), x \in X$, and let $f(x)=\lambda$. There exists $f_{x} \in \mathcal{F}_{x}$ such that $\lambda f_{x} \leq f$. Then we have $\lambda \phi\left(f_{x}\right)=\phi\left(\lambda f_{x}\right) \leq \phi(f)$. This gives us that

$$
f(x)=\lambda=\lambda \phi\left(f_{x}\right)(\varphi(x)) \leq \phi(f)(\varphi(x)) .
$$


Since $\phi^{-1}$ has the same properties as $\phi$, it follows that

$$
\phi^{-1}(\phi(f))\left(\varphi^{-1}(\varphi(x))\right) \geq \phi(f)(\varphi(x)),
$$

that is, we also have $f(x) \geq \phi(f)(\varphi(x))$. Therefore, we obtain $\phi(f)(\varphi(x))=f(x)$ for every $x \in X$ and $0 \leq f \in C(X)$.

We show that $\varphi$ is a homeomorphism. We need only to show that $\varphi$ is continuous. Let $x_{n}$ be a sequence in $X$ converging to the point $x \in X$. Suppose that $\varphi\left(x_{n}\right)$ does not converge to $\varphi(x)$. Then there is a neighbourhood $U$ of $\varphi(x)$ such that $\varphi\left(x_{n}\right) \notin U$ for infinitely many indices. Let $h^{\prime}: X \rightarrow[0,1]$ be a continuous function with support in $U$ such that $h^{\prime}(\varphi(x))=1$. Let $h \in C(X)$ be such that $\phi(h)=h^{\prime}$. Then we have $h\left(x_{n}\right)=h^{\prime}\left(\varphi\left(x_{n}\right)\right)=0$ for infinitely many $n$ 's and this contradicts $h\left(x_{n}\right) \rightarrow h(x)=1$. So, $\varphi$ is a homeomorphism of $X$ and we have $\phi(f)=f \circ \varphi^{-1}$ for every nonnegative $f \in C(X)$.

Finally, for any $f \in C(X)$ and $0 \leq h \in C(X)$ we compute

$$
\sigma\left(\phi(f) \cdot h \circ \varphi^{-1}\right)=\sigma(\phi(f) \phi(h))=\sigma(f h)=\sigma\left(f \circ \varphi^{-1} \cdot h \circ \varphi^{-1}\right)
$$

which gives us that $\phi(f)=f \circ \varphi^{-1}$. This completes the proof.

We turn to the second type of our preserver problems involving involution. We have the following result.

Theorem 6. Let $X$ be a first countable compact Hausdorff space. If $\phi: C(X) \rightarrow$ $C(X)$ is a surjective function with the property that

$$
\sigma(\overline{\phi(f)} \phi(g))=\sigma(\bar{f} g) \quad(f, g \in C(X)),
$$

then there exist a homeomorphism $\varphi: X \rightarrow X$ and a function $\tau \in C(X)$ of modulus 1 such that

$$
\phi(f)(x)=\tau(x) f(\varphi(x)) \quad(x \in X, f \in C(X)) .
$$

Proof. Similarly as in the proof of Theorem 5 one can verify that $\phi$ is injective. Indeed, if $f, g \in C(X)$ are such that $\phi(f)=\phi(g)$, then we have

$$
\sigma(\bar{f} h)=\sigma(\overline{\phi(f)} \phi(h))=\sigma(\overline{\phi(g)} \phi(h))=\sigma(\bar{g} h)
$$

for every $h \in C(X)$ which implies that $f=g$.

Observe that we have $|\phi(1)|^{2}=1$ which implies that $\phi(1)$ is a function of modulus 1. Considering the transformation $f \mapsto \overline{\phi(1)} \phi(f)$, we can and do assume that our function $\phi$ satisfies $\phi(1)=1$. We have

$$
\sigma(\phi(g))=\sigma(\overline{\phi(1)} \phi(g))=\sigma(\overline{1} g)=\sigma(g)
$$

for every $g \in C(X)$. Therefore, $\phi$ is a self-bijection of the set $C_{\mathbb{R}}(X)$ of all real valued continuous functions on $X$ which satisfies

$$
\sigma(\phi(f) \phi(g))=\sigma(f g) \quad\left(f, g \in C_{\mathbb{R}}(X)\right) .
$$

Since, as it turns out from the proof of the previous result, Theorem 5 remains valid for the function algebra $C_{\mathbb{R}}(X)$ as well, we obtain that there is a homeomorphism $\varphi: X \rightarrow X$ such that

$$
\phi(f)=f \circ \varphi \quad\left(f \in C_{\mathbb{R}}(X)\right) .
$$


If $f \in C(X)$, then we have

$$
\sigma(\phi(f) \cdot g \circ \varphi)=\sigma(\phi(f) \phi(g))=\sigma(f g)=\sigma(f \circ \varphi \cdot g \circ \varphi)
$$

for every $g \in C_{\mathbb{R}}(X)$ which yields $\phi(f)=f \circ \varphi$. The proof is complete.

Finally, we present an application of our results. Let $\mathcal{A}$ be a Banach algebra. The transformation $\phi: \mathcal{A} \rightarrow \mathcal{A}$ (no linearity or continuity is assumed) is called a 2local automorphism if for every $x, y \in \mathcal{A}$ there exists an algebra automorphism $\phi_{x, y}$ of $\mathcal{A}$ such that $\phi(x)=\phi_{x, y}(x)$ and $\phi(y)=\phi_{x, y}(y)$. Similarly, the transformation $\psi: \mathcal{A} \rightarrow \mathcal{A}$ is called a 2-local isometry if for every $x, y \in \mathcal{A}$ there exists a surjective linear isometry $\psi_{x, y}$ of $\mathcal{A}$ such that $\psi(x)=\psi_{x, y}(x)$ and $\psi(y)=\psi_{x, y}(y)$. 2-local maps were first studied by Šemrl in [11].

Let $H$ be an infinite dimensional separable Hilbert space. It was proved in [11] that every 2-local automorphism of $B(H)$ is an algebra automorphism of $B(H)$. As for the function algebra $C(X)$ over a compact Hausdorff space $X$, it follows from 5 . 1.2. Theorem] that every 2-local automorphism of $C(X)$ is linear. Hence, applying our result [8, Theorem 2.2], we see that if $X$ is a first countable compact Hausdorff space, then every 2-local automorphism of $C(X)$ is an algebra automorphism.

As for the isometry groups of the above-mentioned algebras, we refer to [7] where we have proved that every 2-local isometry of any $C^{*}$-subalgebra $\mathcal{A}$ of $B(H)$ which contains the ideal of all compact operators and the identity operator is linear. In particular, we obtained that every 2-local isometry of $B(H)$ is a surjective linear isometry of $B(H)$. Unfortunately, we do not know whether the analogue statement is true for $C(X), X$ being a first countable compact Hausdorff space.

Referring back to Šemrl's result on 2-local automorphisms of $B(H)$, examining the proof of [11, Theorem 1], it seems essential that $H$ is a Hilbert space. It is a natural question to ask what can be stated for Banach spaces. It follows from the form of the automorphims of $B(X)$ (every algebra automorphism of $B(X)$ is inner) and Theorem 1 that if $X$ is an infinite dimensional Banach space and $\phi: B(X) \rightarrow$ $B(X)$ is a surjective 2-local automorphism, then $\phi$ is an algebra automorphism of $B(X)$. For an analogous result concerning linear (1-)local automorphisms see [ $\underline{6}$, Theorem 2.1].

\section{REFERENCES}

1. L. Baribeau and T. Ransford, Non-linear spectrum-preserving maps, Bull. London Math. Soc. 32 (2000), 8-14. MR 2000j:15008

2. S.H. Hochwald, Multiplicative maps on matrices that preserve the spectrum, Linear Algebra Appl. 212/213 (1994), 339-351. MR 95h:15051

3. A.A. Jafarian and A.R. Sourour, Spectrum-preserving linear maps, J. Funct. Anal. 66 (1986), 255-261. MR 87m:47011

4. J.C. Hou, Rank-preserving linear maps on B(X), Sci. China Ser. A 32 (1989), 929-940. MR 92b:47052

5. S. Kowalski and Z. Slodkowski, A characterization of multiplicative linear functionals in Banach algebras, Studia Math. 67 (1980), 215-223. MR 82d:46070

6. D.R. Larson and A.R. Sourour, Local derivations and local automorphisms of $B(X)$, in Proc. Sympos. Pure Math. 51, Part 2, Providence, Rhode Island, 1990, 187-194. MR 91k:47106

7. L. Molnár, 2-local isometries of some operator algebras, Proc. Edinb. Math. Soc., to appear.

8. L. Molnár and B. Zalar, Reflexivity of the group of surjective isometries on some Banach spaces, Proc. Edinb. Math. Soc. 42 (1999), 17-36. MR 2000b:47094

9. T. Petek and P. Šemrl, Characterization of Jordan homomorphisms on $M_{n}$ using preserving properties, Linear Algebra Appl. 269 (1998), 33-46. MR 98i:15030 
10. P. Šemrl, Two characterizations of automorphisms on B(X), Studia Math. 105 (1993), 143149. MR 94h:47064

11. P. Šmrl, Local automorphisms and derivations on $B(H)$, Proc. Amer. Math. Soc. 125 (1997), 2677-2680. MR 98e:46082

Institute of Mathematics and Informatics, University of Debrecen, 4010 Debrecen, P.O. Box 12, Hungary

E-mail address: molnarl@math.klte.hu 